\title{
Deciphering Cancer Complexities in Genetically Engineered Mice
}

\author{
K. Simin, ${ }^{*}$ R. Hill ${ }^{\dagger}$ Y. Song, ${ }^{*}$ Q. Zhang, ${ }^{*}$ R. BaSh, ${ }^{*}$ R.D. CARdifF ${ }^{\ddagger}$ C. Yin, ${ }^{*}$ A. XiaO, ${ }^{\text {II }}$ \\ K. MCCARTHY, * AND T. VAN DYKE* \\ *University of North Carolina School of Medicine, Chapel Hill, North Carolina 27599; \\ University of California at Los Angeles, Los Angeles, California 90095; \\ tDepartment of Pathology, University of California, Davis, California 95616
}

\begin{abstract}
Because the $\mathrm{pRb}$ pathway is disrupted in most solid human cancers, we have generated genetically engineered mouse cancer models by inactivating $\mathrm{pRb}$ function in several cell types, including astrocytes and mammary, prostate, ovarian, and brain choroid plexus epithelia. In every case, proliferation and apoptosis are acutely induced, predisposing to malignancy. Cell type dictates the pathways involved in tumor progression. In the astrocytoma model, we developed strategies to induce events in the adult brain, either throughout the tissue or focally. Both K-Ras activation and Pten inactivation play significant roles in progression. In the prostate model, adenocarcinoma progression depends on Pten inactivation. However, nonautonomous induction of p53 in the mesenchyme leads to evolution of both compartments, with p53 loss occurring in the mesenchyme. Thus, studies in these models continue to identify key tumorigenesis mechanisms. Furthermore, we are hopeful that the models will provide useful preclinical systems for diagnostic and therapeutic development.
\end{abstract}

In an effort to model and understand human cancers, we have generated several genetically engineered mouse (GEM) cancer models based on highly penetrant tumor suppressor gene aberrations observed in human tumors. Because the $\mathrm{pRb}$ pathway is disrupted in most solid human cancers, we have inactivated $\mathrm{pRb}$ function cellspecifically in the mouse by transgenic expression of $\mathrm{T}_{121}$, a truncated SV40 T antigen that binds and inactivates $\mathrm{pRb}$ and related proteins $\mathrm{p} 107$ and $\mathrm{p} 130$. In numerous cell types, including mammary (Simin et al. 2004), prostate (Hill et al. 2005a,b), ovarian (C. Yin and T. Van Dyke, unpubl.), and brain choroid plexus (Symonds et al. 1994), epithelia and astrocytes (Xiao et al. 2002), inactivation of $\mathrm{pRb}, \mathrm{p} 107$, and $\mathrm{p} 130$ leads to similar induction of aberrant proliferation and concomitant apoptosis (Fig. 1). In addition, these cellular responses have predisposed to malignancy in each case, producing fully penetrant preclinical models for cancer initiation and progression.

In exploring the genetic basis for tumor progression, we have discovered that the specific pathways involved depend on cell type. Whereas mammary and brain epithelia are similar in that p53 mediates most of the apoptotic response and its loss is the predominant mechanism for tumor progression (Symonds et al. 1994; Lu et al. 2001; Simin et al. 2004), astrocytic and prostate epithelial apoptosis depend on Pten (Xiao et al. 2002; Hill et al. 2005a). Brain and mammary epithelia are also divergent in their requirement for E2F1 for proliferation. In choroid plexus epithelium, proliferation is mediated by E2F1 activity (Pan et al. 1998), in contrast to mammary epithelium, where E2F1 activity is dispensable (M. Sardo and T. Van Dyke, unpubl.). These differences have significant impli-

\footnotetext{
"Present address: Rockefeller University, New York, New York 10021.
}

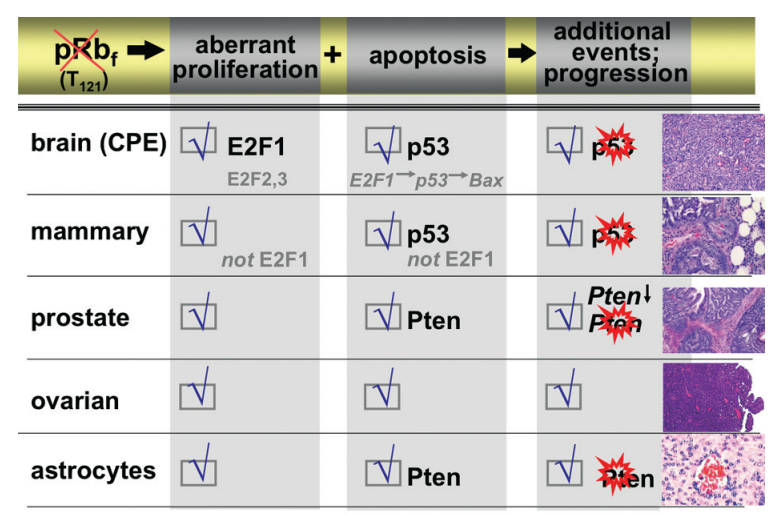

Figure 1. Summary of cancer progression mechanisms. A similar course of events occurs in each system analyzed: $T_{121}$ blocks $\mathrm{pRb}$ activity and causes aberrant proliferation and concomitant increased apoptosis; a selective pressure exists for reduced apoptosis among these neoplastic cells, leading to a subsequent loss of apoptosis and acquisition of other tumor-promoting alterations. However, despite the similarity of events, cells differ in the mechanisms they employ for these functions; brain epithelial cells (CPE) and breast cells rely on p53-mediated apoptosis, whereas astrocytes and prostate epithelial cells depend on Ptenmediated apoptosis. Likewise, whereas E2F1 is required for apoptosis and optimal cellular proliferation in CPE, E2F1 is dispensable in mammary epithelial cells. These differences affect the evolution and biology of each tumor type and may ultimately influence the way we diagnose and treat these cancers.

cations for the mechanisms of tumor progression and the biological behaviors of respective tumor types.

In recent studies, we have examined the complex mechanisms of tumor progression in astrocytic and prostate cancers, including the contribution of multiple Pten-regulated pathways to multiple aspects of tumor progression, such as cell survival, invasion, and angiogenesis (Hill et al. 2005a; Xiao et al. 2005), and of stro- 
mal p53 loss to the progression of prostate tumor (Hill et al. 2005b). Thus, the complex dynamics of tumor evolution are being deciphered for these significant human cancers. Our current efforts to understand these mechanisms are discussed.

\section{MATERIALS AND METHODS}

Mice. The generation and the characterization of $\operatorname{TgGZT}_{121}, \operatorname{Tg} G(\Delta Z) T_{121}$ (Xiao et al. 2002), and $\operatorname{Tg} A P T_{121}$ (Hill et al. 2005a) mice have been described previously. We generated $\operatorname{Tg} G(\Delta Z) T_{121}$ by mating $T g G Z T_{121}$ mice to Tgß-actinCre mice (Lewandoski and Martin 1997) to generate bi-transgenic mice, selecting for pure $\operatorname{Tg} G(\Delta Z) T_{121}$ mice in subsequent generations. Screening of the Pten nullizygous allele has been described previously (Di Cristofano et al. 1998). Characterization and screening of the p53 null allele (Jacks et al. 1994), the conditional Pten allele (Trotman et al. 2003; Xiao et al. 2005), and the LSL$K$-ras ${ }^{G 12 D}$ allele (Jackson et al. 2001) have been described. TgGFAP-CreER mice were constructed and characterized in the McCarthy lab (K. McCarthy et al., unpubl.) and are genotyped using the following parameters: primers are 5'-TGATGAGGTTCGCAAGAACC-3' and 5'-CCATGAGTGAACGAACCTGG-3'. PCR conditions: $94^{\circ} \mathrm{C} 1.5 \mathrm{~min}, 35$ cycles of $94^{\circ} \mathrm{C} 30 \mathrm{sec}, 59^{\circ} \mathrm{C} 1 \mathrm{~min}$, and $72^{\circ} \mathrm{C} 5 \mathrm{~min}$, and finally $72^{\circ} \mathrm{C} 5 \mathrm{~min}$.

Tamoxifen-induced Cre expression. Cre recombinase was activated in TgGFAP-CreER mice by daily i.p. injections of $1 \mathrm{mg}$ of 4-hydroxytamoxifen (4-OHT) over 5 days. Tamoxifen was dissolved in a 1:9 mixture of ethanol and sunflower oil $(100 \mu \mathrm{l})$, facilitated by 10 minute sonication at mid power. Vehicle-only injections served as a control.

Brain histology and immunohistochemistry. Brain tissue was fixed in formalin (10\%) for 20-24 hours and then stored in ethanol (70\%). Paraffin-embedded sections ( $5 \mu \mathrm{m}$ thick) were processed using standard immunohistochemical methods. The antibodies and their dilution were: anti-p-Erk 42/44 (Cell Signaling, 1:50) and antiSV40 Tag (1:200, mouse monoclonal, Oncogene). Briefly, samples were deparaffinized with Histo-Clear (National Diagnostics), then hydrated through a graded ethanol series. Boiling-mediated antigen retrieval was performed using citrate buffer ( $\mathrm{pH}$ 6.0) and microwave heating for 2 minutes at high power followed by $7 \mathrm{~min}$ utes at low power. Endogenous peroxidases were quenched with $\mathrm{H}_{2} \mathrm{O}_{2}(3 \%)$. Immunohistochemistry was performed using the Vectastain ABC kit with the NovaRED (Vector Laboratories, Burlingame, California) peroxidase substrate as chromogen. Samples were counterstained with Harris hematoxylin (Sigma, St. Louis, Missouri). A subset of samples were stained with ABCAP substrate kit (Vector Laboratories) and counterstained with Nuclear Fast Red.

Prostate histopathology and in situ assays. Prostate samples were prepared and analyzed as reported previously (Hill et al. 2005a,b).
Laser capture microdissection and LOH analysis. Laser capture microdissection (LCM) of H\&E-stained sections was performed using a Leica AS LMD (Leica Microsystems Inc., Bannockburn, Illinois) as described previously (Hill et al. 2005b). Briefly, formalin-fixed paraffin-embedded tissue sections were mounted onto Glass Foiled PEN slides (Vashaw Scientific, Atlanta, Georgia). Cells were collected in $50 \mu \mathrm{l}$ of lysis buffer (10 $\mathrm{mm}$ Tris-HCl, $\mathrm{pH} 8.0,1 \%$ Tween-20) with addition of 5 $\mu \mathrm{l}$ of proteinase $\mathrm{K}(100 \mathrm{mg} / \mathrm{ml})$, and incubated at $55^{\circ} \mathrm{C}$ overnight. Proteinase $\mathrm{K}$ was inactivated at $99^{\circ} \mathrm{C}$ for 10 minutes, and 5-10- $\mu 1$ aliquots were used for PCR analysis. The primers for semi-quantitative PCR and quantitative real-time PCR analysis were as described previously (Hill et al. 2005b). The data were analyzed using SDS 2.1 software (Applied Biosystems, Foster City, California) and standard protocols (http://www.appliedbiosystems. com) as previously described (Hill et al. 2005b).

Statistical analysis. Binomial exact test was performed using SAS 9.1 (Cary, North Carolina) to determine whether loss of the wild-type $p 53$ allele was statistically significant in tumor stroma of $\operatorname{TgAPT}_{121} ; \mathrm{p53}^{+/-}$and $\operatorname{TgAPT} T_{121} ; p 53^{+/+}$mice. The probability of random wildtype $p 53$ allele loss in the tumor stroma was arbitrarily set at $1 \%$. However, results remain significant $(p<0.0001)$ even if the probability of random loss is as high as $10 \%$.

\section{RESULTS}

\section{An Astrocytoma Model and New Systems for Inducing $\mathbf{T}_{121}$}

Astrocytoma is the most common primary brain tumor in humans and is rarely amenable to standard therapies. We have engineered mouse models to gain insight to the mechanisms of this poorly understood and often lethal disease, because to fully understand the complexities of such pathologies requires investigation in vivo. The vast majority (70-80\%) of high-grade anaplastic astrocytomas (WHO grade III) and glioblastomas (WHO grade IV, GBM) harbor mutations in the $\mathrm{pRb}$ pathway (Henson et al. 1994; Ueki et al. 1996) in addition to other genetic alterations such as p53 ( 30\%) and PTEN loss-of-function ( $~ 50 \%)$ and EGFR gain-of-function (50\%) mutations (Louis and Cavenee 1997; Maher et al. 2001; Reilly and Jacks 2001). Such high frequencies indicate that these defects are often concurrent with $\mathrm{pRb}$ pathway loss. Therefore, we designed experiments to investigate the combined effects of these perturbations in the defined experimental setting of GEM.

We used a promoter from the human gene encoding glial fibrillary acidic protein $(G F A P)$ to direct $\mathrm{T}_{121}$ expression within the astrocyte lineage of the central nervous system (CNS) (Fig. 2A) (Xiao et al. 2002). However, expression of $\mathrm{T}_{121}$ is dependent on Cre recombinase activity, since a lac $Z$ reporter gene flanked by lox $\mathrm{P}$ sites lies between the promoter and the $T_{121}$ gene $\left(\operatorname{TgGZT} T_{121}\right.$ mice). In $\operatorname{Tg} G(\Delta Z) T_{121}$ (generated after crossing to $\operatorname{Tg} \beta$ actinCre mice; see Materials and Methods) (Lewandoski and Martin 1997), $\mathrm{T}_{121}$ is expressed from embryonic day 
A
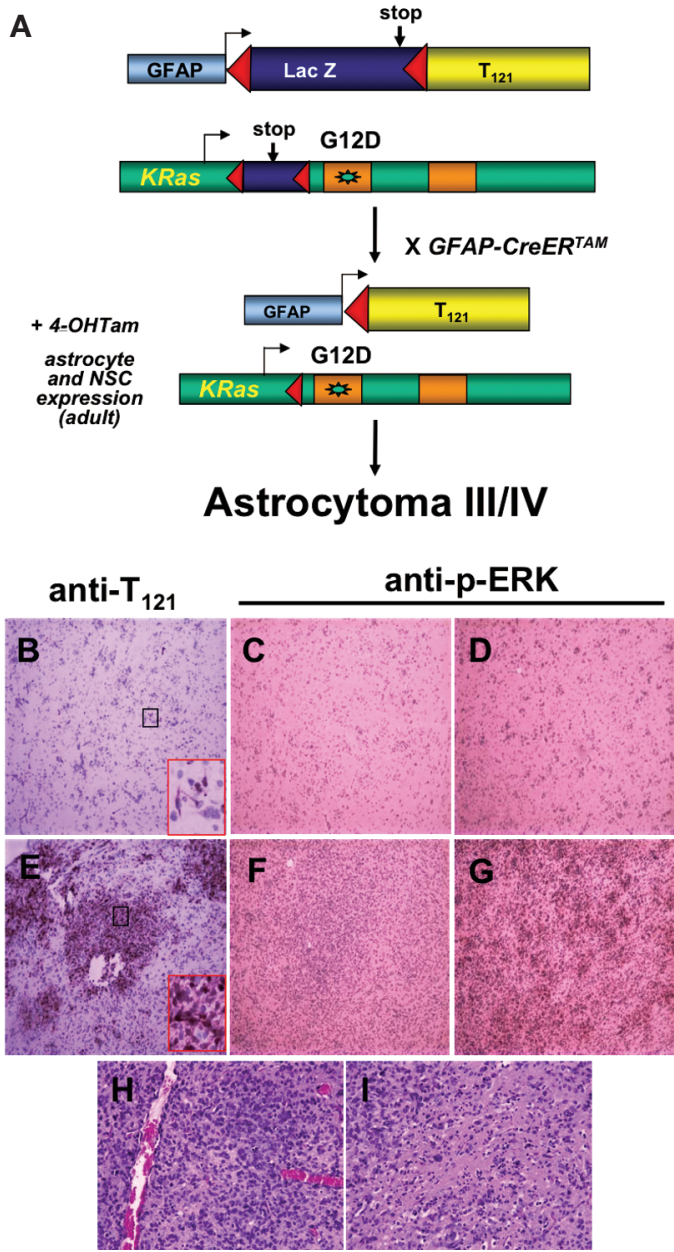

Figure 2. Conditional inactivation of pRb, p107, and p130 combined with Ras activation in adult astrocytes causes progression to high-grade astrocytoma. Two Cre-conditional alleles, encoding $\mathrm{T}_{121}\left(\operatorname{TgGZT}_{121}\right)$ and an activating K-ras point mutant ( $L S L$ $K-$ ras $^{G 12 D}$ ), were used to test the consequences of $\mathrm{pRb}$ function inactivation and Ras activation, respectively, singly and combined $(A)$. Both genes were activated in adult mice at 2-3 months of age using a tamoxifen-inducible Cre recombinase encoded by the GFAP-CreER transgene to avoid confounding developmental effects. Two months post-4-OHT, mice with a combination of $\mathrm{T}_{121}$ and activated K-ras $(E-G)$ show a dramatic expansion of astrocytes that express $\mathrm{T}_{121}(E)$ as compared to mice expressing $\mathrm{T}_{121}$ alone $(B-D)$. Ras-dependent activation of the MAPK pathway is indicated in cortical neoplastic cells by phospho-Erk staining $(G)$ but not in those of the thalamus $(F)$. At 4 months post-4-OHT, both angiogenesis $(H)$ and necrosis are apparent $(I)$ in mice with both $\mathrm{T}_{121}$ and activated K-ras. Samples $B, C, E, F, H$, and $I$ are thalamus; $D$ and $G$ are cortex.

11.5 onward and ultimately results in high-grade astrocytoma in adult mice with $100 \%$ penetrance (Xiao et al. 2002). Although we observed diffuse grade III astrocytomas, none had progressed to malignant grade IV astrocytoma (also called glioblastoma multiforme, or GBM). A secondary phenotype we observed in brains of these mice was an expansion of cells in the subventricular zone (SVZ), a cell population of neuronal lineage and not a glial lineage, which we hypothesize was due to the early expression of GFAP promoter in neuronal progenitor
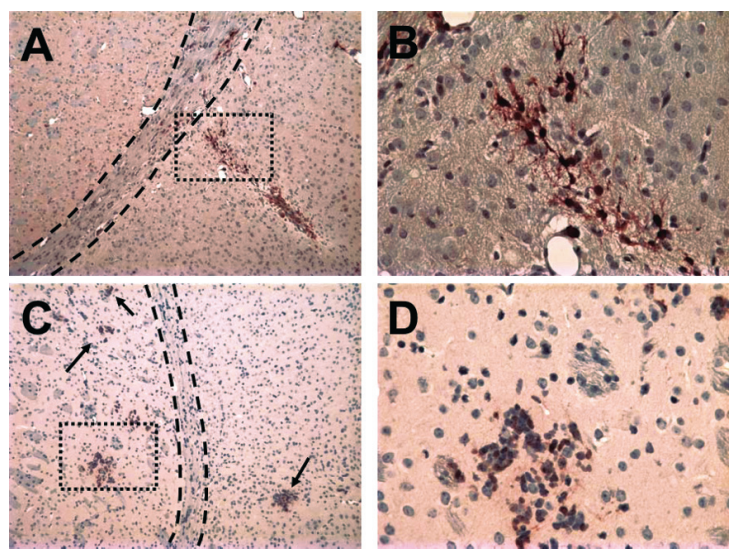

Figure 3. Focal inactivation of $\mathrm{pRb}$ function in adult astrocytes by lentiviral Cre delivery. As an alternative to germ-line transgenesis using tissue-specific promoter elements, we introduced lentivirus harboring a self-excising-Cre construct (unpublished, Tal Kafri, UNC) into the frontal cortex of $T g G Z T_{121}$ transgenic mice via stereotaxic injection to initiate localized tumorigenesis. Nineteen days postinjection, $\mathrm{T}_{121}$ (dark brown stain) can be detected along the needle track (dashed lines) in astrocytic cells (A). Six months postinjection, clusters of astrocytic cells (arrows) can be seen along the injection site $(C)$ and as far as $1500 \mu \mathrm{m}$ away. Magnifications: $A, C, 100 \mathrm{x} ; B, D, 400 \mathrm{x} . B$ and $D$ are higher magnifications of the regions boxed in $A$ and $C$, respectively.

cells. To circumvent the possible effects of $\mathrm{T}_{121}$ activity during CNS development and to better model the human disease, we crossed $T g G Z T_{121}$ mice with $T g G F A P$ CreER mice (K. McCarthy, UNC), such that Cre activity can be controlled by treatment with 4-OHT (Fig. 3A). Indeed, $T_{121}$ was inducible in astrocytes of adult mice (2-3 months of age) and yielded diffuse astrocytoma over time as observed in $\operatorname{Tg} G(\Delta Z) T_{121}$ mice, establishing a valuable inducible model of astrocytoma initiation.

We investigated the possibility that cooperating mutations would synergize with $\mathrm{pRb}$ pathway loss to facilitate tumor progression to more severe grades of disease. Although not frequently mutated, Ras is frequently activated in human high-grade astrocytomas (Bos 1989), likely through amplification or mutation of the epidermal growth factor receptor (EGFR; $\sim 40-60 \%$ of high-grade tumors) or platelet-derived growth factor (PDGF). Thus, Ras activation generally coexists with aberrations of the $\mathrm{pRb}$ pathway. Previous studies showed that overexpression of mutant H-Ras in transgenic astrocytes (Ding et al. 2001), or of activated K-ras in combination with activated Akt (Holland et al. 2000), predisposed GBM-like lesions. We examined the consequences of astrocyte-specific endogenous K-ras activation alone and together with astrocytic $\mathrm{T}_{121}$ expression. Through matings, we generated $T g G Z T_{121} ; T g G F A P-C r e E R, \quad L S L-K$-ras ${ }^{G 12 D /+}$; TgGFAP-CreER, and TgGZT 121 ; LSL-K-ras ${ }^{G 12 D /+}$; TgGFAP-CreER mice (Fig 2A). At 2-3 months of age, Cre activity was induced by treating the mice with 4-OHT (see Materials and Methods) and brains were analyzed at 2 and 4 months postinduction. Activation of Ras alone in astrocytes had no apparent effects up to 4 months post 4-OHT injection (not shown), whereas activation of $\mathrm{T}_{121}$ in astrocytes induced an astrocytic expansion within 2 
months as described above (Fig. 2B). However, a more dramatic phenotype was observed when both $\mathrm{T}_{121}$ and $\mathrm{K}$ ras were activated simultaneously; by 2 months following 4-OHT injections, brains showed features characteristic of human astrocytoma, including peri-neuron and perivascular satellitosis (Fig. 2E). Neoplastic astrocytes were positive for $\mathrm{T}_{121}$, and evidence for Ras-specific activation of the MAPK pathway was demonstrated by phosphoErk IHC. Importantly, phospho-Erk was detectable in the cortex, but not in the thalamus (Fig. 2E, F), suggesting region-specific effects.

Significantly, by 4 months post 4-OHT, angiogenesis (Fig. 2H) and necrosis (Fig. 2I), both properties of GBM, were apparent when both Ras and $\mathrm{T}_{121}$ were activated, but not when either was singly activated (data not shown). We conclude from these results that $\mathrm{pRb}$ pathway loss is sufficient to predispose astrocytes to tumorigenesis, and that additional events, such as Ras activation, are required for astrocytoma tumor progression to GBM.

\section{Somatic Induction of $\mathrm{T}_{121}$ Using Cre-expressing Lentivirus Vectors}

The recombinase-mediated experiments outlined above provide temporal, but not spatial, control of tumor induction. Since human cancers likely evolve from a single cell, initiating tumorigenesis focally rather than in a large cell population may provide a more authentic model of sporadic cancer, mimicking the scenario in which a transformed cell originates within a microenvironment of wild-type cells. As an alternative to germ-line transgenesis using tissue-specific promoter elements, we are developing retroviral approaches to somatically deliver Cre recombinase in adult cells in more limited regions. In one model, lentivirus harboring a self-excising-Cre gene (a kind gift of Tal Kafri, UNC) was introduced into brains of $\operatorname{TgGZT}_{121}$ transgenic mice via stereotaxic injection to initiate local tumorigenesis by expressing $\mathrm{T}_{121}$ in a limited number of astrocytes. One microliter of lentivirus $\left(10^{9}\right.$ $\mathrm{IU} / \mathrm{ml})$ was injected into the cortex of adult $\operatorname{TgGZT}_{121}$ mice $(n=4)$. Nineteen days post injection, $\mathrm{T}_{121}$ was detected by IHC along the needle track in astrocytic cells $(n=2$, Fig. $3 \mathrm{~A}, \mathrm{~B})$. To determine whether local $\mathrm{T}_{121}$ expression was sufficient to generate astrocytoma, the remaining mice $(n=2)$ were aged for 6 months. Despite $\mathrm{T}_{121}$ expression in clusters of astrocytic cells along the injection site (Fig. 3C,D), and as far as $1500 \mu \mathrm{m}$ away, these conditions were insufficient for astrocytoma development. Although we did not observe tumors in this small cohort, the results of this pilot study show promise for this technique; therefore, we continue to explore the consequences of limited regional $\mathrm{pRb}$ pathway inactivation together with additional events such as $K$-ras activation and Pten inactivation, and evaluate its effectiveness as a new paradigm for modeling sporadic cancer.
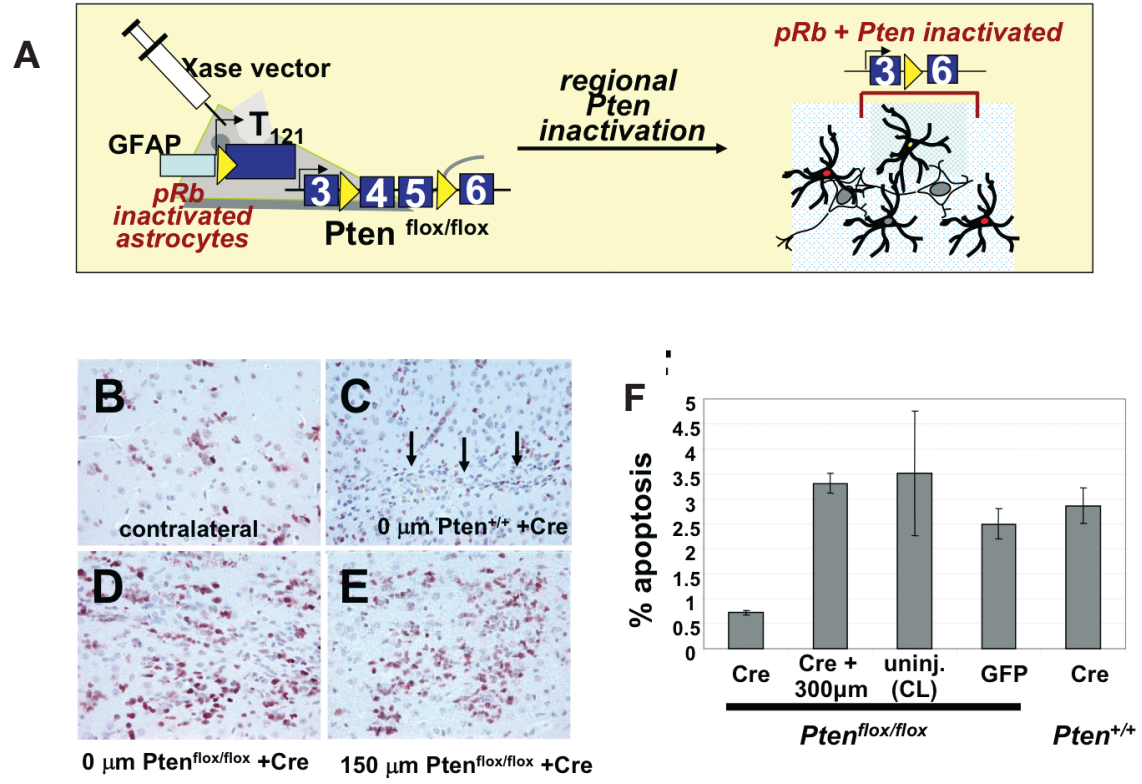

Figure 4. Regional somatic Pten inactivation in $\mathrm{pRb}$-inactivated astrocytes. Somatic delivery of Cre $(A)$ induces accumulation of neoplastic cells. PCNA immunohistochemical staining of brains 5 weeks after retroviral delivery. Viral-Cre delivery to $\operatorname{Tg} G(\Delta Z) T_{121} ;$ Pten $^{\text {loxp/loxp }}$ brains induced dense neoplastic cell foci at the injection sites $(D)$ and in the regions as far as $150 \mu \mathrm{m}$ away $(E)$. The highly dense neoplastic cell foci were not present at the comparable region of the uninjected contralateral hemisphere $(B)$. Focal tumor development was not due to retrovirus infection $\left(\operatorname{Tg} G(\Delta Z) T_{121} ;\right.$ Pten $^{\text {loxp/loxp }}+$ GFP) or Cre protein $\left(T g G(\Delta Z) T_{121} ; P_{t e n}^{+/+}\right.$ + Cre; $C)$ alone, because no such nodules were found in these controls. The only morphologic difference observed between these brains and those not injected (data not shown) is the needle track $\left(C\right.$, arrows). In $\operatorname{Tg} G(\Delta Z) T_{121} ;$ Pten $^{\text {loxp } / l o x p}$ brains, the apoptotic level was decreased in tumor cell clusters near injection sites $(0.69 \pm 0.04 \%$,), compared with contralateral regions $(3.31 \pm 1.24 \%)$ and distant adjacent regions $(300 \mu \mathrm{m}$ from the injection site) where injection tracks were not present $(3.51 \pm 0.20 \%)$. Similar levels of apoptosis were also found at the injected sites of controls $(2.50 \pm 0.53 \%$ and $2.86 \pm 0.30 \%)$. The $\operatorname{Tg} G(\Delta Z) T_{121} ;$ Pten $^{\text {loxp/loxp }}+$ Cre group is compared with each of the other groups; $p<0.05$ is considered statistically significant. (Portions of this figure adapted from Xiao et al. 2005.) 
In a similar experimental approach, we used somatic viral delivery to measure direct and long-term effects of depleting Pten locally in initiated cells already deficient in $\mathrm{pRb}$ pathway function, reminiscent of the clonal changes acquired by human cancers during tumor progression (Xiao et al. 2005). Pten mutations are associated with a significant fraction of human high-grade astrocytomas (30-50\%). We employed an MSCV-Cre viral vector (a kind gift from L. Su, UNC) to assay the effects of Pten loss within initiated tumor cells (Fig. 4A). Because normal astrocytes are non-dividing when terminally differentiated, we reasoned that cells expressing $\mathrm{T}_{121}$ would be more susceptible to retroviral gene delivery because they are highly proliferative at all ages. Indeed, tumor cells along the injection tracks of $\operatorname{Tg} G(\Delta Z) T_{121}$; Pten ${ }^{\text {loxp/loxp }}$ mice had significantly reduced apoptosis $(p<0.05)$, which is evoked by $\mathrm{pRb}$ pathway inactivation, as compared with a distant contralateral region (Fig. 4F). Furthermore, tumor-celldense foci were detected at some distance from the injection tracks in $\operatorname{Tg} G(\Delta Z) T_{121}$; Pten ${ }^{\text {loxp/loxp }}$ brains (Fig. 4E), but not $\operatorname{Tg} G(\Delta Z) T_{121}$; Pten $^{+/+}$brains (Fig. 4C), indicating Pten loss may also facilitate tumor cell invasion.

Using primary astrocytes from $\operatorname{Tg} G(\Delta Z) T_{121}$; $P_{t e n}{ }^{\text {loxp/loxp }}$ newborn mice, we showed that Pten inactivation by adenovirus- $M S C V$-Cre induced invasion through Matrigel. This primary cell model facilitates analysis of pathways involved in tumorigenic properties and should provide a valuable resource for therapeutic target discovery and validation. In preliminary tests using inhibitors, we showed that invasion induced by Pten inactivation was inhibited by both a PI3 kinase inhibitor and a pan protein kinase $\mathrm{C}(\mathrm{PKC})$ inhibitor. Furthermore, treatment of cells with a peptide inhibitor of the atypical PKC- $\zeta$ also significantly suppressed Matrigel invasion (Xiao et al. 2005).

These studies indicate that Pten loss can potentiate multiple tumorigenic properties in neoplastic astrocytes initiated by inactivation of $\mathrm{pRb}$ function and provide both primary cell and animal models for further assessment of the pathways and mechanisms involved. Current studies are exploring the relative contributions of both $K$-ras activation and Pten inactivation to astrocytoma progression using each of the above-described strategies.

\section{Complexity of Tumor Progression in a Model of Prostate Cancer}

A number of useful transgenic models of prostate cancer have provided the means to investigate one of the most common epithelial cancers afflicting men (Greenberg et al. 1994; Yoshidome et al. 1998). However, because these previous strategies employed wild-type SV40 Large T antigen or the full SV40 early region, these models do not permit assessment of the individual contributions of $\mathrm{pRb}$ family loss versus the multiple other concomitant perturbations, some of which have demonstrated roles in tumorigenesis, such as p53 or p300 deficiency, or blocked phosphatase PP2A function. We have shown that inactivation of $\mathrm{pRb}$ function mediated by modified rat probasin promoter-driven expression of $\mathrm{T}_{121}\left(\operatorname{Tg}_{\mathrm{O}} A P T_{121}\right)$ is sufficient for tumor initiation, produces widespread intraepithelial neoplasia (PIN) consisting of high rates of epithelial cell proliferation and apoptosis (Fig. 5B), and ultimately progresses to locally invasive adenocarcinoma

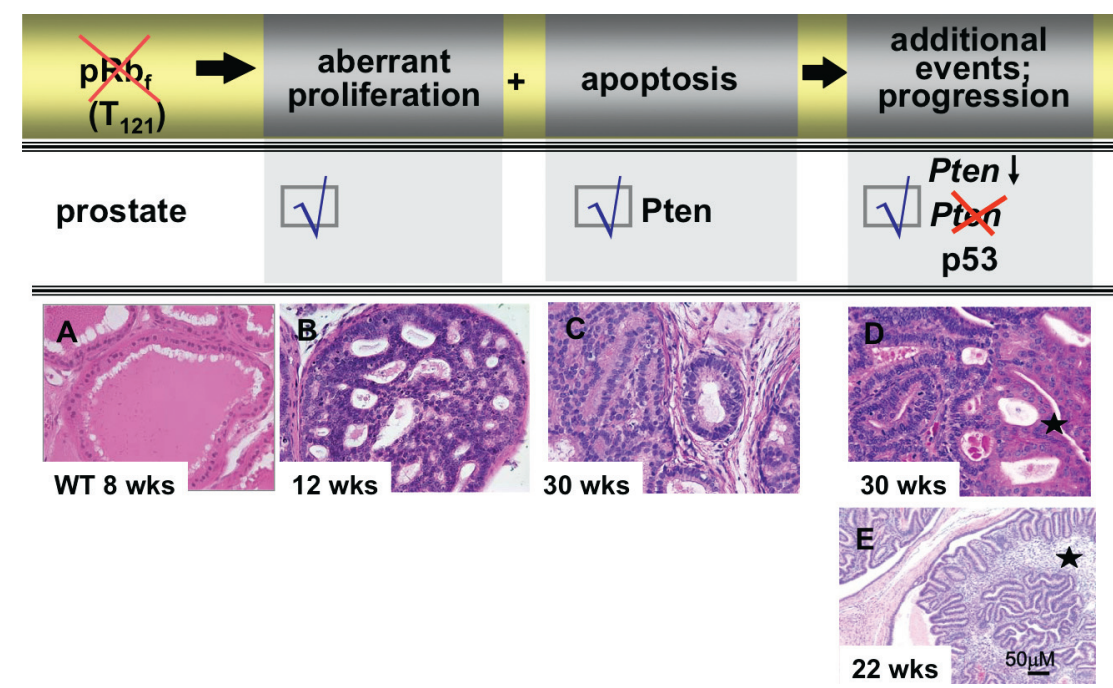

Figure 5. $T g A P T_{121}$ mice develop prostate adenocarcinoma. Inactivation of pRb function leads to aberrant proliferation, and apoptosis is mediated by Pten, not p53. Additional events such as loss of Pten and/or $p 53$ lead to heterogeneous tumor progression. Prostate sections $(5 \mu \mathrm{m})$ shown are from an 8-week-old nontransgenic littermate $(A)$ and $T g A P T_{121}$ mice of different ages $(B, C)$. Normal prostate displays a single layer of luminal cells and a thin fibromuscular stromal cell layer $(A)$. By 12 weeks $(B)$, mouse PIN (mPIN) appears broadly where glands are filled with atypical cells in a cribriform pattern. More advanced lesions are present in prostates of 30 -week-old $\operatorname{Tg} A P T_{121}$ mice, including microinvasive glands with a desmoplastic response and well-differentiated adenocarcinoma $(C)$. Pten heterozygosity accelerates the onset of $\mathrm{mPIN}$ and adenocarcinoma in $T g A P T_{121}$ mice $(D)$. In addition, morphologically distinct areas of adenocarcinoma progression also appear $(D, 30$-week-old, star $)$. p53 heterozygosity does not accelerate the onset of mPIN; however, stromal tumors develop as young as 22 weeks of age $(E)$ and consist of an abundance of stromal cells (star). All sections were stained with hematoxylin and eosin (H\&E). (A-D, Adapted from Hill et al. 2005a; $E$, adapted from Hill et al. 2005b.) 
with no evidence of neuroendocrine tumors (Fig. 5C) (Hill et al. 2005a). Therefore, the $\operatorname{Tg} A P T_{121}$ mouse line provides a fully penetrant mouse model of prostate cancer initiation with which to dissect the mechanisms of tumor progression.

\section{Roles of Pten in Prostate Cancer Suppression}

Because apoptosis induced by $\mathrm{pRb}$ functional loss is mediated through different mechanisms in different cell types, we examined the pathway(s) used by $\operatorname{Tg} A P T_{121}$ prostates with a genetic approach. Using the TUNEL assay, we quantified prostate apoptosis levels in 8-week-old mice of the following genotypes: wild type, $\operatorname{Tg}_{A P} T_{121}$, $\operatorname{TgAPT}_{121} ;$ Pten $^{+/}, \operatorname{TgAPT}_{121} ; \mathrm{p5}^{+-}$, and $\operatorname{TgAPT}_{121}$; $p 53^{-/}$. In $T g A P T_{121}$ prostates, hemizygosity or nullizygosity of $p 53$ caused no reduction in the apoptotic index, whereas hemizygosity at Pten caused a 50\% reduction in the apoptotic index accelerating progression to adenocarcinomas with heterogeneous composition (Fig. 6A). Het-
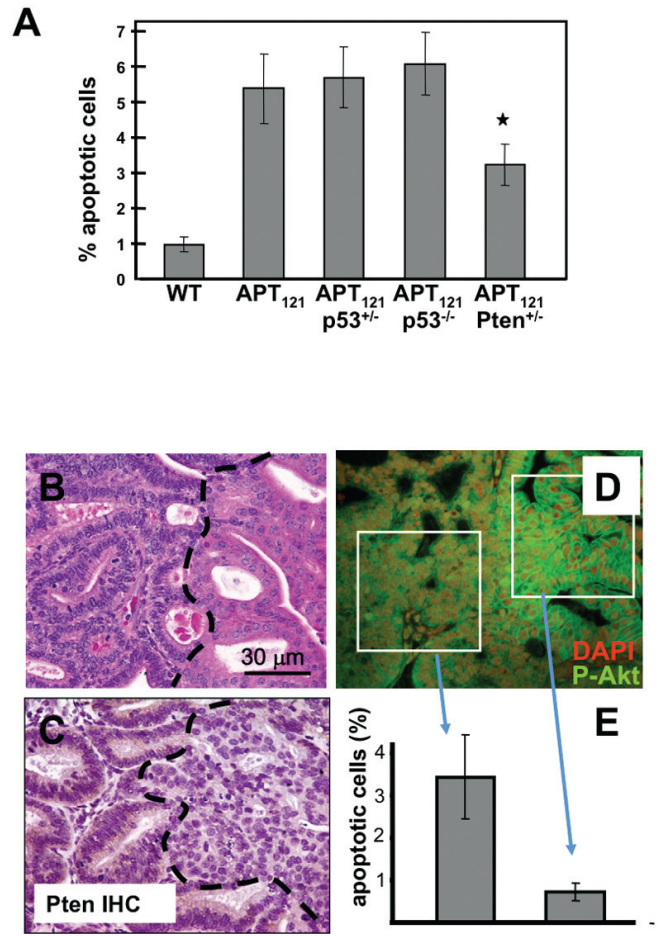

Figure 6. Pten mediates apoptosis in $T g A P T_{121}$ prostate, and its loss contributes to focal tumor progression. $(A)$ Prostates from 8-

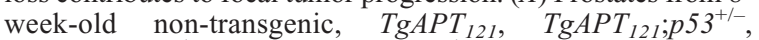
$\operatorname{TgAPT}_{121} ; p 53^{-/-}$, and $T g A P T_{121} ;$ Pten $^{+--}$mice were analyzed for apoptosis levels quantified by calculating the number of TUNELpositive cells (brown) as a percentage of the total (methyl green). $n=4$ for each genotype. $(B)$ A representative H\&E-stained section shows a transition where focal adenocarcinoma develops from neoplastic areas with back-to-back gland structure. $(C) \mathrm{Im}-$ munohistochemistry for Pten (red/brown) shows loss of cytoplasmic staining in regions of focal progression. In such regions of Pten loss, cells express membrane-bound p-Akt staining ( $D$; green). (E) Apoptosis assay was performed via TUNEL in those p-Akt expressed and nonexpressed regions (white boxes). p-Akt expressed cells, indicating loss of Pten, have further reduction of apoptosis. (Adapted from Hill et al. 2005a.) erogeneity is associated with concurrent Pten haploinsufficiency and selective focal tumor progression identifiable by concomitant Pten loss (Fig. 6C), the appearance of membrane-bound phospho-Akt (p-Akt)(Fig. 6D), a further reduction in apoptosis levels (Fig. 7E), and distinct morphology (Fig. 6B).

\section{Unexpected Roles for p53 in Epithelial/Mesenchymal Interactions}

Although p53 is not involved in epithelial apoptosis in $\operatorname{Tg} A P T_{121}$ mice, we found that it plays a critical role in tumor evolution. $T_{121}$ expression in prostate epithelium resulted in p53 induction in both epithelial and mesenchymal compartments (Hill et al. 2005b). p53 appears to

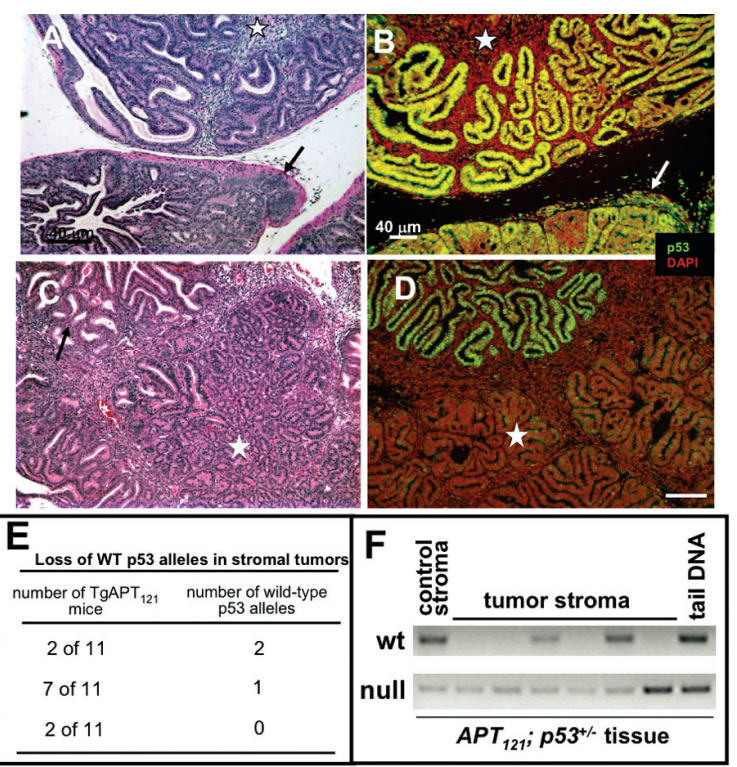

Figure 7. Stromal p53 loss in $T g A P T_{121} ; p 53^{+/-}$and $\operatorname{Tg} A P T_{121}$ tumors. Serial sections of an emerging stromal tumor in $T g A P T_{121} ; p^{2} 3^{+/-}$mice with expanding mesenchyme (star) and adjacent mPIN (arrow) are shown ( $A$; H\&E) and ( $B$; p53 IF). p53 expression is lost in the tumor mesenchyme $(B$, star $)$ and retained in the epithelium in addition to the mPIN-associated stroma (arrow). A total of $18 A P T_{121} ; p 53^{+/-}$mice with stromal tumors were examined, and all were found to have lost p53 expression in the stroma. Epithelial p53 loss subsequent to stromal p53 loss compounds heterogeneous tumor progression. In H\&E-

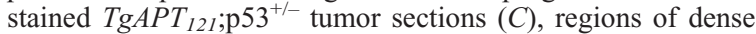
epithelial cell growth morphologically distinct from surrounding epithelium (arrows) grow in small back-to-back circular glands (star). IF for p53 (yellow) shows that such regions no longer express p53. Representative tumors from 7-month-old $T g A P T_{121} ; p 53^{+-1}$ mice are shown $(D)$. PCR analysis on stromal DNA extracted from laser-captured microdissected (LCM) samples in $T g A P T_{121} ; p 53^{+/-}$mice showed that stromal loss of wildtype $p 53$ allele occurred in four of six tumors $(F)$. Similar stromal loss of $\mathrm{p} 53$ expression is observed in stromal tumors arising in $T g A P T_{121} ; p 53^{+/+}$mice $(n=8)$. Real-time quantitative PCR was performed on stromal DNA extracted from LCM samples to determine the status of wild-type $p 53$ alleles in stromal tumors or tissues of $T g A P T_{121}$ mice. LCM muscle samples were used as controls. Among 11 stromal tumor samples from 11 distinct animals, 7 showed loss of 1 wild-type allele of $p 53,2$ showed loss of both alleles of $p 53$, and 2 retained both wild-type alleles. (Portions of this figure adapted from Hill et al. 2005b.) 
suppress fibroblast proliferation in the stroma, since these cells proliferated in a $T g A P T_{121}$ p53-deficient, but not wild-type or heterozygous, background (Hill et al. 2005b). Indeed, all $\operatorname{Tg}_{A P} T_{121} ; p 53^{-/-}$and $\operatorname{Tg} A P T_{121}$; $p 53^{+/-}$(Fig. 5E) male mice developed large tumors composed of extensive stroma, a phenotype that develops most rapidly in the $p 53$ null background. Tumor fibroblasts of the $p 53$ heterozygous background no longer expressed $p 53$ (Fig. 7B), and laser capture microdissectionPCR analysis showed that the wild-type $p 53$ allele was selectively lost in this compartment in most tumors (Fig. $7 \mathrm{~B}, \mathrm{~F})$. Similar stromal loss of $p 53$ expression is observed in stromal tumors arising in $\operatorname{TgAPT} T_{121} ; p 53^{+/+}$mice $(n=$ $8)$. Real-time quantitative PCR on stromal DNA extracted from LCM samples showed that among 11 stromal tumor samples from 11 distinct animals, 7 lost 1 wild-type allele of $p 53,2$ lost both alleles of $p 53$, and 2 retained both wild-type alleles (Fig. 7E) (Hill et al. $2005 b)$. Although the epithelium within these tumors initially retained p53 expression (Fig. 7B), focal regions subsequently lost p53 expression as well (Fig. 7D), suggesting an as yet unknown function of p53 as tumor suppressor in this compartment. These studies demonstrate that non-cell-autonomous responses to epithelial tumorigenic lesions can create a microenvironment in which selective genetic changes occur in non-tumor cells, causing coevolution of multiple cell types within the tumor. Current studies are focused on identifying the p53-inductive signal(s) from initiated epithelium to the mesenchyme and on the mechanisms of p53 tumor suppression in multiple prostate compartments.

\section{CONCLUSIONS}

By exploiting $T_{121}$ to inactivate $\mathrm{pRb}$ and its related family members, we have explored the requirement of $\mathrm{pRb}$ tumor suppression in glial cells and multiple epithelial cell types, including brain (CPE), prostate, and breast. This has been a powerful approach to generate highly penetrant mouse models of cancer that are reflective of human tumors in which the $\mathrm{pRb}$ pathway is perturbed and that evolve through the accumulation of additional genetic or epigenetic aberrations. We have dissected some of the genetic pathways contributing to tumor progression both by combining the initiated transgenic models with other engineered germ-line mutations and by analyzing the natural evolution of progressing tumors. Through these experiments, we have identified differences in tumor suppressor mechanisms invoked by varying cell types. More specifically, in each of the systems studied, there is a similar course of events: $T_{121}$ expression causes aberrant proliferation and concomitant increased levels of apoptosis, resulting in a strong selective pressure for reduced apoptosis. Tumors progress when a major tumor suppressor is inactivated, resulting in reduced apoptosis, but also in additional tumor-promoting functions. In response to $\mathrm{pRb}$ function loss, CPE and breast cells rely on p53-mediated apoptosis, whereas astrocytes and prostate epithelium depend on Pten activity. These differences significantly affect the dynamics of cancer evolution, and therefore may have wide-ranging implications in the interpretation of diagnostic tumor mutation analysis and in the development of appropriately targeted therapies.

Because $T_{121}$ so effectively evokes illicit cellular proliferation and apoptosis, we have until recently relied on gene regulatory sequences to direct $T_{121}$ expression only in adult cells to avoid secondary developmental defects. We have further refined our strategy by engineering conditional alleles using Cre-LoxP technology to more finely restrict the timing and location of $\mathrm{T}_{121}$ expression. Building on this conditional approach, we now also employ retroviral vectors to manipulate more limited cell populations to investigate the consequences of focal events, both in tumor initiation and in events that may contribute to tumor progression. This paradigm more closely mimics natural spontaneous tumor evolution. Combined, these strategies facilitate the analysis of acute consequences to specific genetic lesions, both intrinsic and extrinsic, and the dissection of natural events in the evolution of specific tumors. We are hopeful that the GEM models produced in these studies will also provide useful preclinical systems for diagnostic and therapeutic development.

\section{ACKNOWLEDGMENTS}

We thank Jim Pipas (University of Pittsburgh) for generating and characterizing the $\mathrm{T}_{121}$ (d11137) mutant $\mathrm{T}$ antigen and for sharing reagents and insightful discussions in the early stages of this work. We thank Hua Wu and Lucy Lu for expert technical assistance in generating transgenic mice, and also Drew Fogarty, Ginger Muse, Daniel Roth, Anne Wolthusen, and Chunyu Yang for colony maintenance and mouse genotyping. We also thank Tal Kafri and Lishan Su (University of North Carolina) for providing Cre-expressing viral vectors, and Tyler Jacks (Massachusetts Institute of Technology) for providing $L S L-K-$ ras $^{G 12 D}$ mice. This work was supported by grants from the National Cancer Institute (U01CA84314 and R01-CA046283 to T.V.D. and U01CA84294 to R.D.C.), the Susan B. Komen Foundation (T.V.D.), the Department of Defense (breast and prostate cancer programs; T.V.D.), the Brain Tumor Society (T.V.D.), and the Goldhirsh Foundation (T.V.D.).

\section{REFERENCES}

Bos J.L. 1989. ras oncogenes in human cancer: A review. Cancer Res. 49: 4682.

Di Cristofano A., Pesce B., Cordon-Cardo C., and Pandolfi P.P. 1998. Pten is essential for embryonic development and tumour suppression. Nat. Genet. 19: 348.

Ding H., Roncari L., Shannon P., Wu X., Lau N., Karaskova J., Gutmann D.H., Squire J.A., Nagy A., and Guha A. 2001. Astrocyte-specific expression of activated p21-ras results in malignant astrocytoma formation in a transgenic mouse model of human gliomas. Cancer Res. 61: 3826.

Greenberg N.M., DeMayo F.J., Sheppard P.C., Barrios R., Lebovitz R., Finegold M., Angelopoulou R., Dodd J.G., Duckworth M.L., and Rosen J.M., et al. 1994. The rat probasin gene promoter directs hormonally and developmentally regulated expression of a heterologous gene specifically to the prostate in transgenic mice. Mol. Endocrinol. 8: 230. 
Henson J.W., Schnitker B.L., Correa K.M., von Deimling A., Fassbender F., Xu H.J., Benedict W.F., Yandell D.W., and Louis D.N. 1994. The retinoblastoma gene is involved in malignant progression of astrocytomas. Ann. Neurol. 36: 714.

Hill R., Song Y., Cardiff R.D., and Van Dyke T. 2005a. Heterogeneous tumor evolution initiated by loss of $\mathrm{pRb}$ function in a preclinical prostate cancer model. Cancer Res. 65: 10243.

. 2005b. Selective evolution of stromal mesenchyme with p53 loss in response to epithelial tumorigenesis. Cell 123: 1001.

Holland E.C., Celestino J., Dai C., Schaefer L., Sawaya R.E., and Fuller G.N. 2000. Combined activation of Ras and Akt in neural progenitors induces glioblastoma formation in mice. Nat. Genet. 25: 55.

Jacks T., Shih T.S., Schmitt E.M., Bronson R.T., Bernards A., and Weinberg R.A. 1994. Tumour predisposition in mice heterozygous for a targeted mutation in Nf1. Nat. Genet. 7: 353.

Jackson E.L., Willis N., Mercer K., Bronson R.T., Crowley D., Montoya R., Jacks T., and Tuveson D.A. 2001. Analysis of lung tumor initiation and progression using conditional expression of oncogenic K-ras. Genes Dev. 15: 3243.

Lewandoski M. and Martin G.R. 1997. Cre-mediated chromosome loss in mice. Nat. Genet. 17: 223

Louis D.N. and Cavenee W.K. 1997. Molecular biology of central nervous system tumors. In Cancer: Principles and practice of oncology (ed. V.T. DeVita et al.), p. 2013. LippincottRaven, Philadelphia, Pennsylvania.

Lu X., Magrane G., Yin C., Louis D.N., Gray J., and Van Dyke T. 2001. Selective inactivation of p53 facilitates mouse epithelial tumor progression without chromosomal instability. Mol. Cell. Biol. 21: 6017.

Maher E.A., Furnari F.B., Bachoo R.M., Rowitch D.H., Louis D.N., Cavenee W.K., and DePinho R.A. 2001. Malignant glioma: Genetics and biology of a grave matter. Genes Dev. 15: 1311 .
Pan H., Yin C., Dyson N., Harlow E., Yamasaki L., and Van Dyke T. 1998. A key role for E2F1 in p53-dependent apoptosis and cell division within developing tumors. Cell 2: 283.

Reilly K.M. and Jacks T. 2001. Genetically engineered mouse models of astrocytoma: GEMs in the rough (review)? Semin. Cancer Biol. 11: 177.

Simin K., Wu H., Lu L., Pinkel D., Albertson D., Cardiff R.D., and Van Dyke T. 2004. pRb inactivation in mammary cells reveals common mechanisms for tumor initiation and progression in divergent epithelia. PLoS Biol. 2: E22.

Symonds H., Krall L., Remington L., Saenz-Robles M., Lowe S., Jacks T., and Van Dyke T. 1994. p53-dependent apoptosis suppresses tumor growth and progression in vivo. Cell 78: 703.

Trotman L.C., Niki M., Dotan Z.A., Koutcher J.A., Cristofano A.D., Xiao A., Khoo A.S., Roy-Burman P., Greenberg N.M., Dyke T.V., Cordon-Cardo C., and Pandolfi P. 2003. Pten dose dictates cancer progression in the prostate. PLoS Biol. 1: E59.

Ueki K., Ono Y., Henson J.W., Efird J.T., von Deimling A., and Louis D.N. 1996. CDKN2/p16 or RB alterations occur in the majority of glioblastomas and are inversely correlated. Cancer Res. 56: 150

Xiao A., Wu H., Pandolfi P.P., Louis D.N., and Van Dyke T. 2002. Astrocyte inactivation of the $\mathrm{pRb}$ pathway predisposes mice to malignant astrocytoma development that is accelerated by PTEN mutation. Cancer Cell 1: 157.

Xiao A., Yin C., Yang C., Di Cristofano A., Pandolfi P.P., and Van Dyke T. 2005. Somatic induction of Pten loss in a preclinical astrocytoma model reveals major roles in disease progression and avenues for target discovery and validation. Cancer Res. 65: 5172.

Yoshidome K., Shibata M.A., Maroulakou I.G., Liu M.L., Jorcyk C.L., Gold L.G., Welch V.N., and Green J.E. 1998. Genetic alterations in the development of mammary and prostate cancer in the C3(1)/Tag transgenic mouse model. Int. J. Oncol. 12: 449. 


\section{$8_{8}^{\infty} \mathrm{CSH} \&$ Cold Spring Harbor Symposia SYMPOSIA on Quantitative Biology}

\section{Deciphering Cancer Complexities in Genetically Engineered Mice}

K. SIMIN, R. HILL, Y. SONG, et al.

Cold Spring Harb Symp Quant Biol 2005 70: 283-290

Access the most recent version at doi:10.1101/sqb.2005.70.038

References This article cites 22 articles, 8 of which can be accessed free at:

http://symposium.cshlp.org/content/70/283.full.html\#ref-list-1

License

Email Alerting Receive free email alerts when new articles cite this article - sign up in

Service the box at the top right corner of the article or click here. 